\title{
Barrieren im Sozialraum - ein Kommentar
}

\author{
Markus Schäfers
}

I. Einführung

II. Teilhabeförderliche Umwelt schaffen

III. Unterstützungssysteme reformieren

IV. Positives Bild von Behinderung in der Öffentlichkeit vermitteln

V. Literatur

\section{Einführung}

Wie lassen sich teilhabeförderliche Umwelten schaffen? Moderne fachliche und sozialpolitische Konzepte richten ihren Blick - stärker als in der Vergangenheit - auf die Umwelten, in denen Menschen mit Behinderung leben und ihren Alltag zu bewältigen versuchen. Damit wird den sozialen Einflussgrößen bei der Entstehung und Ausprägung von Behinderung größerer Stellenwert eingeräumt. Konkrete Umwelten umfassen nicht nur die baulich-materielle Umgebung, sondern auch Aspekte wie die Zugänglichkeit von Informationen, die Einstellung und das Verhalten von Mitbürgerinnen und Mitbürgern gegenüber Menschen mit Behinderung oder die Beschäftigungspolitik von Arbeitgebern.

Insofern erscheint es vielversprechend, bei der Frage danach, wie sich die Möglichkeitsräume von Menschen mit Behinderung zur gesellschaftlichen Teilhabe erweitern lassen, bei den vielfältigen gesellschaftlich geprägten Umweltbarrieren anzusetzen. Welche Zielrichtungen, Strategien und Konzepte erscheinen dabei zukunftsweisend?

Ich möchte von drei Thesen ausgehen, um zentrale Herausforderungen, die mit dem Thema „Inklusionsbarrieren“ zusammenhängen, zu identifizieren und da, wo es möglich erscheint, Ansätze zur Lösung und Überwindung folgender Herausforderungen abzuleiten:

1. Die „Inklusionsfähigkeit“ der Gesellschaft ist begrenzt

2. Traditionelle Hilfesysteme werden als ineffektiv beurteilt und als Instanzen, die selbst exkludierend wirken 
3. Das Bild von Behinderung in der Öffentlichkeit festigt einstellungsbedingte Barrieren

\section{Teilhabeförderliche Umwelt schaffen}

Sozialraumorientierung, Regionalplanung, örtliche Teilhabeplanung - all diese Konzepte und Instrumente laufen darauf hinaus, eine inklusive bzw. teilhabeförderliche Umwelt zu schaffen, in denen Menschen mit unterschiedlichen Voraussetzungen und Bedürfnissen ein Leben nach ihren Vorstellungen führen können ${ }^{1}$.

Inwiefern sozialräumlich angelegte Konzepte dabei der Schlüssel zum Erfolg sein können, wird insbesondere in der Behindertenhilfe kontrovers diskutiert: Von manchen wird Sozialraumorientierung als inklusionsförderliche Innovation betrachtet, das die Verbesonderung von Hilfen zu überwinden verspricht ${ }^{2}$, von anderen als Sparmodell disqualifiziert, das ein Recht des Einzelnen auf bedarfsgerechte Unterstützung zu unterlaufen versucht ${ }^{3}$.

In der Vergangenheit hat sich die traditionelle Behindertenhilfe ambivalent verhalten: Einerseits hat sie die begrenzte „Inklusionsfähigkeit“ der gesellschaftlichen Regelsysteme kritisiert und die damit einhergehenden Exklusionsmechanismen im Bildungsbereich, auf dem Arbeitsmarkt, im kulturellen Leben usw. Andererseits hat sie sich selbst als Sondersystem entwickelt, die Regelsysteme von ihren Aufgaben entlastet und so paradoxerweise dazu beigetragen, dass Menschen mit Behinderung aus gesellschaftlich relevanten Zusammenhängen der Lebensführung weitgehend ausgeschlossen und „unsichtbar" wurden ${ }^{4}$.

Mit der Zielbestimmung, gesellschaftliche Teilhabe zu fördern, gilt es nun für die Behindertenhilfe, sich stärker in der Gesellschaft zu verorten, mit ihren Aktivitäten und Kompetenzen das Gemeinwesen mitzugestalten und vor Ort präventiv und unterstützend zu wirken.

Sozialraumorientiert zu handeln, fallübergreifend zu denken und die Sozialräume, in denen sich Menschen bewegen, als vornehmliches Handlungsfeld zu begreifen, entspricht nicht der Tradition und Kultur der Behindertenhilfe und ist nicht durch rechtliche und finanzielle Bedingungen unterlegt. Das Leistungsrecht ist durch das Bedarfsdeckungsprinzip des Einzelnen geprägt und durch einen individuellen Rechtsanspruch auf Hilfe. Wie lässt sich so sozialräumliche Arbeit organisieren und nachhaltig finanzieren?

1 Ausführlich zu einer örtlichen Teilhabeplanung Welti, S. 87 in diesem Band.

2 Hinte, Innovation oder Depression - Zum Dilemma der Diskussion um Sozialraumorientierung, Teilhabe 2/2012, S. 66.

3 Dahme/Wohlfahrt, Der Sozialraum als Rettungsanker des Sozialstaats mit antikapitalistischer Durchschlagskraft?, Teilhabe 2/2012, S. 69.

4 Wacker/Wansing/Schäfers, Personenbezogene Unterstützung und Lebensqualität, Teilhabe mit einem Persönlichen Budget, Wiesbaden 2005, S. 17. 
Leistungsanbieter haben nur dann einen (wirtschaftlichen) Vorteil, wenn möglichst viele Hilfefälle produziert und bearbeitet werden. Sozialraumorientiertes Handeln hingegen versucht, eine Typisierung und Unterscheidung zwischen hilfebedürftig/nicht hilfebedürftig möglichst zu vermeiden.

Zwar gibt es auch in der Behindertenhilfe bereits jetzt schon wegweisende und erfolgreiche Konzepte sozialräumlichen Handelns, indem beispielsweise in einer Region Arbeitsplätze für Menschen mit Behinderung geschaffen werden durch Netzwerkarbeit, Patenmodelle und individuelles Job-Coaching 5 . Diese Ansätze arbeiten im Moment gegen die bestehenden rechtlichen und finanziellen Strukturen und werden nicht von ihnen gestützt. So wird es schwer sein, sie voll zum Tragen kommen zu lassen und weiter zu verbreiten.

\section{Unterstützungssysteme reformieren}

In der sozialpolitischen Diskussion um die Weiterentwicklung der Unterstützungssysteme, insbesondere im Kontext der Reform der Eingliederungshilfe, steht aber nicht der Sozialraum im Zentrum der Überlegungen, sondern der Begriff der „Personzentrierung“. Personzentrierte Hilfe wird verstanden und (verkürzt) dekliniert als Fokussierung auf den Bedarf des Einzelnen, Bewilligung der Leistungen unabhängig vom Ort und der Form der Leistungserbringung (Aufhebung der Trennung zwischen ambulant und stationär), Unterscheidung der Fachleistung von existenzsichernden Leistungen und ein personenbezogenes Fallmanagement des Sozialhilfeträgers 6 .

Die Umstellung von der Angebots- zur Personzentrierung ist programmatisch ein Schritt in die richtige Richtung, aber nicht ausreichend. Der Wirkkraft einer ausschließlichen Beschäftigung mit dem Einzelnen, seinen Kompetenzen und Defiziten sind Grenzen gesetzt; Probleme werden weiterhin vorwiegend im Individuum verortet, die Umweltbedingungen werden so nicht hinreichend in den Blick genommen. Dazu braucht es einen ergänzenden Blick auf die Infrastruktur und die Gegebenheiten des Sozialraums.

Wenn es um die Frage geht, in welche Richtung das Wohlfahrtssystem gelenkt werden soll, wird unter dem Stichwort „Personzentrierung“ als wesentliche Stellschraube immer wieder das Wunsch- und Wahlrecht des Leistungsberechtigten hervorgehoben. Hier herrscht erstaunliche Einigkeit bei allen Akteuren, von der Selbsthilfe, den Leistungsanbietern bis zu den Leistungsträgern.

5 Basener/Häußler, Bamberg bewegt, Integration in den Arbeitsmarkt: Eine Region wird aktiv, Hamburg 2008.

6 ASMK - Bund-Länder-Arbeitsgruppe „Weiterentwicklung der Eingliederungshilfe für Menschen mit Behinderungen“ der ASMK, Eckpunkte für die Reformgesetzgebung Eingliederungshilfe im SGB XII, 2009, abrufbar unter http://www.alle-inklusive.de/wp-content/uploads/2009/12/ASMK2009-Anlage-1-Eckpunkte-Eingliederungshilfe.pdf. 
Das Wunsch- und Wahlrecht zu stärken und Auswahlmöglichkeiten zu schaffen, ist sicherlich richtig und wichtig, dagegen lässt sich kaum etwas glaubhaft einwenden. Allerdings besteht die Gefahr, dass die Bedeutung des Wunsch- und Wahlrechts im Kontext dieser Diskussion überformt wird. Sie darf nicht hochstilisiert werden zum einzigen Regulativ für die Weiterentwicklung der Unterstützungssysteme insgesamt.

Kritisch lässt sich einwenden, dass sich das Wunsch- und Wahlrecht zur Legitimation jedweder Angebotsentwicklung missbrauchen lässt - salopp gesagt nach dem Motto: „Hauptsache, es bestehen Alternativen“. Gerade das, was als Alternativen zur Verfügung steht, präsentiert sich aber immer schon so, wie es gesellschaftlich-historisch entstanden ist (z. B. in Bezug auf die stationäre und ambulante Angebotsstruktur in einer Region). Zudem ist das Wunsch- und Wahlrecht selbst zeitlich und kulturell eingebunden ${ }^{7}$; was Menschen mit Behinderung und ihre Familien heute wollen, ist Ausdruck dessen, was sie in der Vergangenheit erfahren haben und in der Gegenwart bilanzieren. Zugleich stellt sich die Frage, ob das Wunsch- und Wahlrecht repräsentativ und damit geeignet ist, zu einem Ausgleich unterschiedlicher Interessen beizutragen: Schließlich ist davon auszugehen, dass nicht alle Menschen mit Behinderung unabhängig von der Schwere ihrer Beeinträchtigung in gleichem Maße die Chance haben, bei Entscheidungen, die sie betreffen, gehört zu werden und ihr Recht durchzusetzen, z. B. wenn es um die Auswahl und Gestaltung von Wohn- und Betreuungsformen geht ${ }^{8}$.

Die Gesamtverantwortung, teilhabeförderliche Bedingungen zu schaffen und geeignete Unterstützungssysteme bereitzustellen, ist eine staatliche. Um diese Entwicklungsaufgabe meistern zu können, ist eine Vorstellung davon erforderlich, wie das Wohlfahrtssystem in den nächsten Jahren und Jahrzehnten gestaltet sein muss, um die Bedarfe zukünftiger Nutzerinnen und Nutzer der Unterstützungsleistungen zu decken und gesellschaftliche Herausforderungen bewältigen zu können. In diesem Zusammenhang kommt dem Wunsch- und Wahlrecht sicherlich eine gewisse Bedeutung zu, aber diese Verantwortung lässt sich nicht an den Einzelnen und sein Wunsch- und Wahlrecht delegieren.

\section{Positives Bild von Behinderung in der Öffentlichkeit vermitteln}

Eine Neuorientierung des Hilfesystems allein wird nicht ausreichend sein, um teilhabeförderliche Umwelten zu schaffen. Teilhabe beruht ganz wesentlich auf sozialen Austauschprozessen, dazu sind gelingende Anerkennungsprozesse erforderlich ${ }^{9}$. Hier wird die Begrenztheit professionellen Handelns deutlich. Allerdings kann es durchaus fachli-

7 Wocken, Elternwahlrecht!? Über Dienstbarkeit, Endlichkeit und Widersinn des Elternwillens, Vierteljahresschrift für Heilpädagogik und ihre Nachbargebiete 3/2010, S. 186.

8 Dazu Wansing, S. 69 in diesem Band.

9 Dazu Dederich, S. 61 in diesem Band. 
che Aufgabe sein, günstige Voraussetzungen für gelingende Anerkennungsprozesse zu schaffen. Dies ist jedoch insofern widersprüchlich, als Unterstützungsleistungen für bestimmte Personengruppen die Andersartigkeit der Adressaten markieren können und so zur Verstärkung des Problems beitragen, dessen Lösung sie eigentlich intendieren.

Ein Ansatzpunkt kann darin liegen, das öffentliche Bild von Behinderung, das im Allgemeinen auf festgefahrenen Stereotypen basiert, in positiver Weise zu verstören. Dadurch lässt sich u. U. die feste Grenzziehung zwischen behindert/nicht behindert aufbrechen. Ein Beispiel hierfür ist das bürgerschaftliche Engagement von Menschen mit Behinderung, die sich selbst im Gemeinwesen ehrenamtlich für andere Menschen oder für die Ziele einer Organisation einsetzen, z. B. in Tafel-Projekten, im Naturschutz oder in der Freiwilligen Feuerwehr ${ }^{10}$. Dadurch werden sie nicht mehr ausschließlich in der Rolle des Hilfeempfängers gesehen, sondern als wertvolle Mitbürger, die auch etwas für die Gesellschaft leisten können.

\section{Literatur}

ASMK - Bund-Länder-Arbeitsgruppe „Weiterentwicklung der Eingliederungshilfe für Menschen mit Behinderungen" der ASMK, Eckpunkte für die Reformgesetzgebung Eingliederungshilfe im SGB XII, 2009, http://www.alle-inklusive.de/wp-content/uploads/2009/12/ ASMK-2009-Anlage-1-Eckpunkte-Eingliederungshilfe.pdf (abgerufen am 07.10.2012).

Basener, Dieter/Häußler, Silke, Bamberg bewegt, Integration in den Arbeitsmarkt: Eine Region wird aktiv, Hamburg 2008.

Dahme, Heinz-Jürgen/Wohlfahrt, Norbert, Der Sozialraum als Rettungsanker des Sozialstaats mit antikapitalistischer Durchschlagskraft?, Teilhabe 51 (2) 2012, S. 69.

Hinte, Wolfgang, Innovation oder Depression - Zum Dilemma der Diskussion um Sozialraumorientierung, Teilhabe 51 (2) 2012, S. 66.

Wacker, Elisabeth/Wansing, Gudrun/Schäfers, Markus, Personenbezogene Unterstützung und Lebensqualität, Teilhabe mit einem Persönlichen Budget, Wiesbaden 2005.

Wocken, Hans, Elternwahlrecht!? Über Dienstbarkeit, Endlichkeit und Widersinn des Elternwillens, Vierteljahresschrift für Heilpädagogik und ihre Nachbargebiete 79 (3) 2010, S. 186.

10 Vgl. www.lebenshilfe-aktiv.de. 


\title{
Inklusion im Sozialraum aus Sicht des Deutschen Vereins
}

\author{
Andreas Kuhn
}

I. Inklusion im Sozialraum aus Sicht des Deutschen Vereins

$\begin{array}{ll}\text { II. Soziale Inklusion } & 108\end{array}$

III. Barriere Wertschätzung? 111

IV. Literatur 114

\section{Inklusion im Sozialraum aus Sicht des Deutschen Vereins}

Die UN-Behindertenrechtskonvention hat dazu beigetragen, dass auch im Deutschen Verein das Thema Inklusion intensiv diskutiert wird und Papiere beschlossen wurden, die beschreiben, wie Inklusion zu verwirklichen ist: ein Diskussionspapier zu inklusiver Bildung, Eckpunkte für einen inklusiven Sozialraum und Empfehlungen zu einer örtlichen Teilhabeplanung für ein inklusives Gemeinwesen.

Auch zum Sozialraum hat sich der Deutsche Verein in praktischer Absicht geäußert ${ }^{1}$. Soziale Räume sind als - politisch oder statistisch definierte - Gebiete im Gemeinwesen zu verstehen, als Wohnquartiere und Stadtteile, in denen die soziale Integration der darin lebenden Bewohner und Bewohnerinnen stattfindet. Soziale Räume sind Gebiete im Gemeinwesen, die auch heute die Sozialisation, die persönliche Entwicklung, die Lebensbedingungen und die Lebensweise der darin aufwachsenden und lebenden Individuen beeinflussen ${ }^{2}$. In welchem Ausmaß, ist natürlich individuell unterschiedlich. Generell gilt: Je geringer die Mobilität, desto wichtiger ist der so definierte Sozialraum. Ein zweiter Einflussfaktor ist die residentielle Segregation insbesondere von ethnischreligiösen Gruppen.

Inklusion und Sozialraum in Zusammenhang gebracht haben die Arbeits- und Sozialminister auf ihrer Konferenz (ASMK) im November 2009. Die Konferenz stellt fest:

1 Und unterscheidet sich damit deutlich von den Debatten, die in Deutschland über den Begriff des Sozialen Raums geführt wurden; vgl. z.B. Kessel u.a. (Hrsg.), Handbuch Sozialraum, Wiesbaden 2005.

2 Eckpunkte des Deutschen Vereins zur sozialräumlichen Ausgestaltung kommunalen Handelns, NDV 9/2008, S. 377 ff. 